Trayectorias Universitarias, 6(10), e023, 2020

ISSN 2469-0090 | https://doi.org/10.24215/24690090e023

https://revistas.unlp.edu.ar/TrayectoriasUniversitarias

Universidad Nacional de La Plata

La Plata | Buenos Aires | Argentina

\title{
Una experiencia de educación en emergencia sanitaria: de la presencialidad al entorno virtual
}

\section{An experience of education in health emergency: from face-} to-face to the virtual environment

\author{
Peralta Minini, Verónica \\ https://orcid.org/0000-0002-7938-3723 \\ veritominini@gmail.com \\ Facultad de Ciencias Politicas y Sociales I UNCuyo \\ | Argentina
}

\author{
Musatto, Carolina Alejandra \\ https://orcid.org/0000-0003-2891-144X \\ musattocarolina@gmail.com \\ Facultad de Ciencias Politicas y Sociales I UNCuyo \\ | Argentina
}

\section{RESUMEN}

A través del desarrollo de la siguiente experiencia como docentes, buscamos realizar una descripción y visibilizar aspectos para análisis sobre la modificación y complejidad de los procesos de enseñanza-aprendizaje en el contexto de emergencia sanitaria por Covid-19, que actualmente atraviesa a Argentina y a otros países del mundo.

Frente a este momento tan complejo, el distanciamiento social obligatorio, pone en cuestión las estrategias de enseñanza-aprendizaje tal y como han sido planteadas desde la creación de la educación formal, donde los/as estudiantes, concurren a un espacio físico (el establecimiento educativo) para poder adquirir y construir conocimientos. Es así que desde el corriente año, las normativas impuestas por la emergencia, han implicado que los/as docentes adecuemos los espacios curriculares en los que nos desempeñamos, a las nuevas tecnologías, debiendo repensar no sólo las estrategias a implementar, si no la dificultad de acceso de algunos sujetos a los espacios virtuales.

En este sentido, las condiciones socio-económicas presentes, demuestran que no todos los/as estudiantes poseen el acceso a servicios o tecnología que permita un proceso sostenido en el tiempo de educación virtual.

A su vez, identificamos la necesidad por parte de las instituciones educativas, de implementar mayores recursos institucionales para poder abordar dicha complejidad, fomentando mayor acompañamiento a la diversidad de situaciones que poseen los/as estudiantes.

Es a través de esta narración de nuestra experiencia, que desarrollamos el proceso de transformación, de una estructura presencial a una virtual, abarcando distintos medios y plataformas, y además pensando futuras estrategias, tendientes a promover la permanencia y el avance académico.

PALABRAS CLAVE

entorno,

virtual,

estudiantes, emergencia 
KEY WORDS

environment, virtual, students, emergency

\section{ABSTRACT}

Through the development of the following experience as teachers, we seek to make a description and make visible aspects for analysis on the modification and complexity of the teaching-learning processes in the context of health emergency by Covid-19, which currently crosses Argentina and others countries of the world.

Faced with this complex moment, compulsory social distancing calls into question the teaching-learning strategies as they have been proposed since the creation of formal education, where students attend a physical space (the educational establishment ) to be able to acquire and build knowledge. Thus, since the current year, the regulations imposed by the emergency have implied that teachers adapt the curricular spaces in which we work to new technologies, having to rethink not only the strategies to be implemented, but also the difficulty of access of some subjects to virtual spaces.

In this sense, the current socio-economic conditions show that not all students have access to services or technology that allows a sustained process in virtual education time. At the same time, we identified the need for educational institutions to implement greater institutional resources in order to deal with this complexity, promoting greater accompaniment to the diversity of situations that students have.

It is through this narration of our experience that we develop the transformation process, from a face-to-face structure to a virtual one, encompassing different media and platforms, and also thinking about future strategies, aimed at promoting permanence and academic advancement. 


\section{PRESENTACIÓN}

A través de la siguiente narración, situada en la Universidad Nacional de Cuyo, Provincia de Mendoza, daremos cuenta de nuestra experiencia de enseñanza y aprendizaje en la cátedra "Trabajo Social III: Abordaje Comunitario y Grupal - Desarrollo Local" y sus transformaciones, teniendo en cuenta dos periodos de tiempo caracterizados por entornos de aprendizaje diferentes, debido a los distintos contextos en que hemos desarrollado esta experiencia.

El primer periodo de tiempo, relata la forma de cursado presencial y metodología de evaluación, del año 2019, donde los/as estudiantes continuaban con la estructura de enseñanza presencial, debiendo asistir al establecimiento educativo. Mientras que en el segundo periodo de tiempo que relatamos, aconteciendo durante el corriente año, la presencialidad y su cotidianidad se han visto interrumpidas por la emergencia sanitaria, poniendo en cuestión formas tradicionales de enseñanza, y generando con ello, la búsqueda e implementación de nuevos espacios para brindarle a los/as estudiantes las herramientas teórico-metodológicas de aprendizaje.

Frente a lo desarrollado, se busca generar un análisis, que posibilite repensar las prácticas docentes y estudiantiles, ya que -consideramos- actualmente nos encontramos atravesando un proceso de reconfiguración.

\section{DESCRIPCIÓN DE LA EXPERIENCIA}

Nuestra experiencia tiene lugar en la Facultad de Ciencias Políticas y Sociales, perteneciente a la Universidad Nacional de Cuyo (UNCuyo), donde nos desempeñamos como docentes, formando parte del espacio curricular "Trabajo Social III: Abordaje Comunitario y Grupal - Desarrollo Local", que forma parte del diseño curricular de la Licenciatura en Trabajo Social.

Dicho espacio curricular, se encuentra enmarcado en el Plan de Estu- 
dios de 1999, dentro del "Núcleo III: Fundamentos Teórico-Metodológicos de Trabajo Social", a partir del cual, se busca un análisis histórico y actual de los fundamentos teóricos del Trabajo Social como disciplina, relacionando la construcción de su campo problemático y la cuestión social en la articulación con la intervención profesional.

En cuanto a la especificidad de la asignatura, Trabajo Social III: Abordaje Comunitario y Grupal - Desarrollo local, posee como objetivo que las/los estudiantes comprendan la pertinencia de la intervención profesional en el ámbito comunitario y los procesos históricos por los que ha atravesado el Trabajo Social en el devenir de sus intervenciones en lo comunitario. Dicho recorrido histórico del quehacer de la profesión en ese ámbito, ha tenido estrecha vinculación con el contexto social y político de cada época. Al mismo tiempo ese recorrido histórico permite comprender la relevancia que cobran tres categorías conceptuales: Sujetos, Territorio y Políticas Públicas, que actúan como estructurantes de la intervención profesional en el ámbito comunitario.

Se requiere por tanto una visión compleja, dinámica y contextualizada de la intervención comunitaria que es la que la Asignatura se propone proveer a fin de que el/la estudiante pueda interpretar la trama comunitaria desde la cotidianidad de los sujetos, en un espacio territorial dado, con el fin de construir estrategias de intervención ligadas a la particularidad de las condiciones concretas en que se desarrolle dicha intervención, teniendo como lectura central las políticas públicas vigentes y sus relaciones con los territorios.

Desde el plan de estudios y la metodología de trabajo, históricamente el cursado de la Lic en Trabajo Social y por consecuencia la cátedra de Trabajo Social III, se ha desarrollado a través de la presencialidad, en el espacio físico de la Facultad de Ciencias Políticas y Sociales (FCPyS).

De este modo, en el año 2019, como en años anteriores, el cursado de la asignatura se desarrolló en el tercer año de la carrera, en el primer cuatrimestre, con una carga horaria total de 90 hs. y 6 hs. semanales. La propuesta pedagógica para el desarrollo de la Asignatura, se fundó en una perspectiva constructivista del proceso educativo, en base a la cual los procesos y metodologías para la enseñanza aprendizaje se apoyaron en dispositivos que en las clases presenciales combinaron momentos de: exposición docente; análisis de situaciones prácticas; reconocimiento y recuperación de conocimientos previos por parte de los/as estudiantes; lectura, reflexión y debate sobre material bibliográfico; expresión del pensamiento autónomo y elaboración de opinión fundada.

A su vez, el desarrollo de las clases presenciales se apoyó en la utilización de la Cátedra virtual mediante la cual, los/as estudiantes accedían al programa de la cátedra, a informaciones y agenda, material y/o consignas para trabajos prácticos, guías de clase y bibliografía obligatoria por unidad.

Para el desarrollo de su carácter presencial, en la asignatura se llevaron a cabo los siguientes instrumentos de evaluación: 
- Dos exámenes parciales (correspondientes a la Unidades 1 y 2, y Unidades 3 y 4 respectivamente) de carácter obligatorio e individual. Con formato escrito semiestructurado. Cada examen contaba con una instancia recuperatoria y su aprobación es condición indispensable para obtener la regularidad en la materia. Asimismo, la aprobación en primera instancia de al menos uno de los dos exámenes parciales, permitía acceder al sistema de promoción sin examen final.

- Un trabajo práctico obligatorio y grupal, con la finalidad articular los contenidos teóricos y prácticos de la asignatura y la asistencia a un taller transversal realizado en conjunto con otras cátedras del mismo año y cuatrimestre de cursado.

-Un coloquio final integrador: para quienes alcanzaban las condiciones de acreditación y/o promoción sin examen final. De carácter grupal, oral y de calificación individual.

- Examen final: para quienes optaran por acreditación y/o promoción con examen final o no alcancen las condiciones para la promoción sin examen final. De carácter individual, oral y en las fechas estipuladas en el calendario académico de la facultad.

Asimismo, a comienzos de febrero del corriente año, las actividades educativas de la Universidad y por consecuencia de la asignatura, se desarrollaron con normalidad y con su carácter presencial, desarrollándose así, la creación del programa y agenda-cronograma de temas y días de clases. Continuando con la metodología/estrategia de enseñanza - aprendizaje en base a la presencialidad de los/as estudiantes al dictado de clase y consultas en el edificio de la facultad.

Es así como en el corriente año, luego de las mesas de examen de febrero-marzo y de la presentación de los programas para su debida evaluación por las autoridades académicas, se vieron interrumpidas y modificadas las actividades presenciales, ya que el día 15 de marzo, el Poder Ejecutivo Nacional comunicó que al día siguiente comenzaba una suspensión de clases presenciales en todo el país, aunque con asistencia docente y administrativa en establecimientos educativos de diferentes niveles. Esta medida fue tomada como consecuencia de la propagación de casos de Covid-19 en diferentes países del mundo, y la aparición de casos en nuestro país. Cinco días después, el Presidente Alberto Fernández comunicó al país, la medida de aislamiento social (físico), preventivo y obligatorio (A.S.P.O.) que comenzaría a regir en todo el territorio nacional a partir del 20 de marzo.

Ante esa situación, la FCPyS de la UNCuyo postergó el inicio de clases (que inicialmente sería el 16 de marzo) al día 01 de abril, con formato adaptado a los entornos virtuales disponibles.

En la cátedra en la que formamos parte, Trabajo Social III, Ilevamos a cabo durante esas dos semanas previas al nuevo inicio de cursado, un proceso de planificación y adaptación para dar comienzo al cursado de 
la materia, de forma virtual, sin saber cuánto tiempo duraría el A.S.P.O. y por tanto, el dictado de la asignatura en el entorno virtual.

\section{En la cátedra en la que formamos parte, Trabajo Social III, Ile- vamos a cabo durante esas dos semanas previas al nuevo ini- cio de cursado, un proceso de planificación y adaptación para dar comienzo al cursado de la materia, de forma virtual, sin saber cuánto tiempo duraría el A.S.P.O. y por tanto, el dictado de la asignatura en el entorno virtual.}

Cabe tener en cuenta que para definir un entorno virtual de aprendizaje, hemos tomado los aportes videográficos que realiza Manuel Area Moreira (2015), quien lo caracteriza como un espacio en línea (en plataformas virtuales y/o redes sociales) que está organizado (no es espontáneo) y facilita al estudiante desarrollar experiencias/acciones de aprendizaje.

En este sentido, nos parece fundamental hacer alusión al carácter "organizado" que requiere un espacio virtual, teniendo en cuenta que por primera vez, en el marco del contexto de emergencia sanitaria que estamos atravesando, desde el equipo de cátedra nos vimos en la necesidad de organizar -en un lapso de tiempo reducido- el espacio virtual para el dictado exclusivo de la materia, con posibilidad de que durante este cuatrimestre (y por lo tanto, en la totalidad del cursado de la misma), sea el único entorno en donde se realice dicho cursado, en caso de no retomar antes del mes de julio a la presencialidad.

De esta manera, en pos de organizar el entorno virtual de aprendizaje del que dispondrían las/os estudiantes a partir de abril del corriente, es que empezamos en el equipo de cátedra una serie de encuentros virtuales internos a través de distintos medios (Ilamadas y videollamadas grupales por Messenger y Whatsapp, Ilamadas telefónicas), con el fin de planificar y diseñar aspectos centrales que nos permitieran comenzar el dictado de la materia en un entorno virtual.

En primer lugar, realizamos algunas actualizaciones (del Programa 2020, ejercitación introductoria y material de presentación de la materia) en la principal plataforma de la que disponemos para el dictado de la cátedra: Plataforma de Educación a Distancia Uncuvirtual. Además, creamos un correo electrónico de la materia con la finalidad de tenerlo como apoyo para que las/os estudiantes puedan entregar actividades evaluativas por ese medio en caso que la plataforma virtual presentara inconvenientes.

Por otra parte, tomamos la decisión en el equipo de cátedra, de crear otros espacios de apoyo para la difusión de contenidos e información y la interacción con las/os estudiantes: un grupo cerrado de Facebook y una cuenta de Instagram. Cabe agregar que una vez comenzado el cursado, las/os ayudantes estudiantes que participan en la cátedra, 
crearon y comenzaron a administrar un grupo de Whatsapp con las/ os estudiantes.

Compartimos a continuación algunas imágenes que elaboramos desde el equipo de cátedra, previo al inicio del cursado, y que difundimos en distintos espacios y medios (plataforma Uncuvirtual, grupo cerrado de Facebook en donde se empezó a agregar estudiantes, e Instagram):

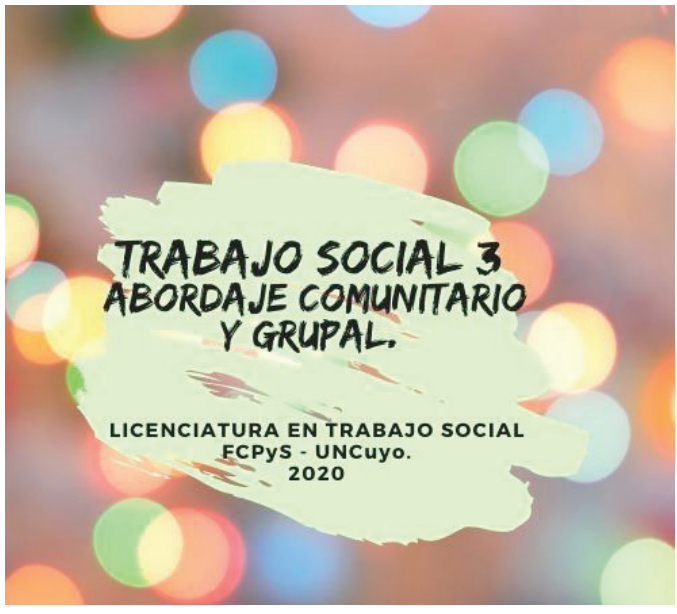

Ilustración 1 (Elaboración equipo de cátedra)

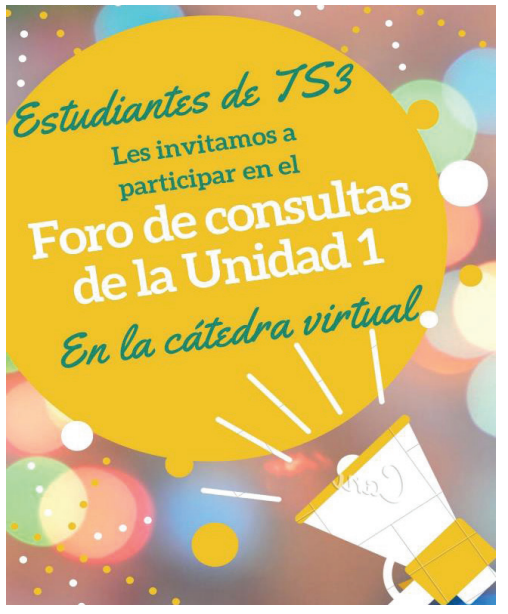

Ilustración 2 (Elaboración equipo de cátedra)

Luego de iniciar el cursado, las/os estudiantes han completado un Formulario Google que elaboramos desde el equipo, para conformar un listado provisorio de la cohorte 2020, ya que aún no contamos con listados oficiales, dado que se postergó en la facultad la reinscripción anual y a materias, habiendo finalizado el 8 de mayo. Además, a través del formulario mencionado, relevamos algunos datos de conectividad y disponibilidad de dispositivos del estudiantado.

En este sentido, cabe destacar que en una cohorte de 126 estudiantes (que han completado el formulario hasta el día 9 de mayo del corriente), 8 de ellas/os no disponen de internet, mientras que 31 disponen sólo de datos móviles, y el porcentaje restante sí tiene wifi. Esto complejiza la posibilidad de pensar en un dictado virtual de la asignatura, y plantea interrogantes sobre futuras medidas que deberán tomarse desde la unidad académica para acompañar las trayectorias y el avance académico de quienes no puedan acceder a los entornos virtuales en el presente cuatrimestre. Los porcentajes de esta variable pueden ser observados en el siguiente gráfico, arrojado por el Formulario Google:

cabe destacar que en una cohorte de 126 estudiantes (que han completado el formulario hasta el día 9 de mayo del corriente), 8 de ellas/os no disponen de internet, mientras que 31 disponen sólo de datos móviles, y el porcentaje restante sí tiene wifi. 
¿Disponés de internet?

123 respuestas

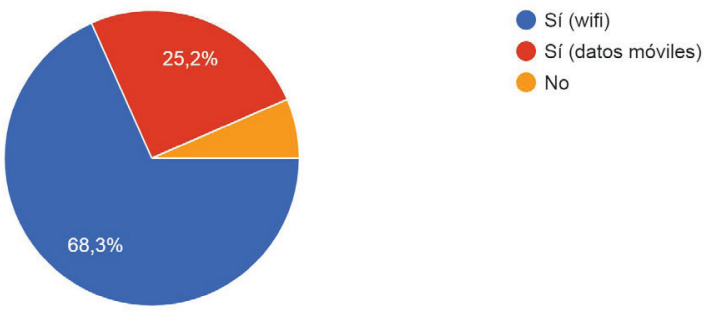

Gráfico 1

Además, otro factor a tener en cuenta es que en la cohorte inscripta, si bien 71 estudiantes cuentan con dispositivos tales como PC, netbook o notebook para llevar a cabo el cursado virtual, otras/os 49 estudiantes sólo cuentan con teléfono celular, y 3 disponen de tablet. Este factor puede ser relevante en tanto que no es la misma visiblidad de textos, páginas web y capacidad en el uso, la que tienen los primeros dispositivos mencionados, en comparación con celulares y tablets, y esto podría impactar -o no- de alguna forma en la continuidad del cursado y/o en la realización de las actividades evaluativas. Estos datos se encuentran graficados a través del Formulario Google de la siguiente manera:

¿De qué dispositivo/s disponés para conectarte en la plataforma virtual? 123 respuestas

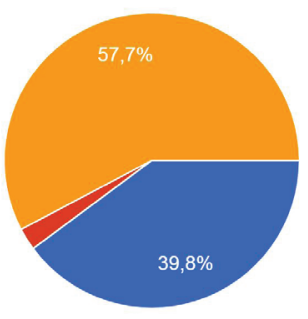

Gráfico 2

Por otra parte, en lo que respecta a las actividades realizadas en el marco del cursado en entorno virtual, hemos implementado la realización de videos y transmisiones en vivo en el grupo de Facebook, que además son editados y subidos a Youtube por el equipo de cátedra, lo que nos permite compartirlos con las estudiantes no sólo por Facebook (como actividad sincrónica, ya que es en directo) sino además en la Uncuvirtual a través de enlace. En estos videos, las profesoras titular y adjunta explican y responden consultas sobre los principales temas de la materia, mientras que el jefe y la jefa de trabajos prácticos, explicamos las consignas y características generales de los trabajos prácticos evaluativos. 
en lo que respecta a las actividades realizadas en el marco del cursado en entorno virtual, hemos implementado la realización de videos y transmisiones en vivo en el grupo de Facebook, que además son editados y subidos a Youtube por el equipo de cátedra, lo que nos permite compartirlos con las estudiantes no sólo por Facebook (como actividad sincrónica, ya que es en directo) sino además en la Uncuvirtual a través de enlace.

También realizamos por cada una de las cinco unidades de la materia, un Foro de Consultas de la Unidad, que se encuentra disponible en la Uncuvirtual, en donde estudiantes realizan consultas referidas a los temas y/o las actividades evaluativas de cada unidad y el equipo docente responde en horarios de consulta.

Como espacio de interacción activa entre docentes y estudiantes, distribuidos en comisiones, hemos implementado además, salas en el grupo de Facebook para hacer devoluciones generales de Trabajos Prácticos, además de las devoluciones específicas que enviamos a cada estudiante o pareja autora de los trabajos prácticos correspondientes. Otro espacio de interacción con las/os estudiantes es la mensajería privada de la Uncuvirtual, a través de la cual también respondemos desde el equipo consultas particulares.

Cabe tener en cuenta que las actividades de intercambio y consulta mencionadas en los párrafos anteriores, no tienen carácter obligatorio ni evaluativo para las/os estudiantes, sino que buscan contribuir a un desarrollo del cursado que permita la interacción y el vínculo entre estudiantes y docentes, así como una explicación más acabada de los temas.

En cuanto a las actividades evaluativas, hemos decidido desde el equipo de cátedra proponer a las/os estudiantes:

- Para regularizar la materia: aprobación de dos trabajos prácticos evaluativos (en primera instancia o en recuperatorio). Luego deberán presentarse a mesas de examen para acreditar la materia.

- Para aprobar la materia por evaluación continua sin examen final: aprobar cinco trabajos prácticos evaluativos (en primera instancia o recuperatorio), es decir, un trabajo práctico por cada una de las cinco unidades, más un trabajo final integrador al finalizar el cuatrimestre.

De este modo, se prevé en el segundo semestre del corriente ciclo, habiendo terminado el dictado de la materia, brindar espacios de consulta personalizados a estudiantes que no hayan podido sostener el cursado en línea de la misma, así como quienes hayan quedado en condición de regular (sin aprobarla por promocionalidad) y deban acreditarla mediante examen final. 
Al mismo tiempo, y para finalizar la descripción de estas transformaciones que estamos atravesando, nos encontramos realizando consultas por videollamadas de Whatsapp y Messenger con estudiantes que cursaron la asignatura en años anteriores y planifican rendirla en mesas de examen.

\section{CONCLUSIONES}

A través del presente trabajo, hemos realizado una descripción y análisis de distintos aspectos vinculados al proceso de enseñanza-aprendizaje que se lleva a cabo en la asignatura "Trabajo Social III: Abordaje Comunitario y Grupal - Desarrollo Local", de la carrera de Licenciatura en Trabajo Social (Facultad de Ciencias Políticas y Sociales, UNCuyo), teniendo en cuenta el contexto actual de emergencia sanitaria que nos atraviesa a nivel local, nacional, regional y mundial.

Como puede identificarse en el relato que realizamos de nuestra experiencia, las modificaciones que desde el equipo de cátedra y el estudiantado estamos afrontando, nos implican la capacitación, exploración y utilización de nuevas metodologías en espacios virtuales de enseñanza-aprendizaje y evaluación, así como de dictado de los temas y la construcción colectiva de los conocimientos.

De este modo, las transformaciones que estamos atravesando en materia educativa nos llevan a retomar los aportes de Luz Osorio Gómez (2011), quien plantea que el surgimiento de Internet y de la Web, así como los avances de las últimas décadas en tecnologías de la información y la comunicación, permiten pensar la necesidad y/o posibilidad de una integración entre modelos de educación presencial y modelos de educación virtual. En este sentido, la autora expresa que los ambientes híbridos de aprendizaje, constituyen una alternativa en la que convergen dos modos de aprendizaje: el cara a cara o presencial y el distribuido o en línea, y que una de las ventajas de construir e implementar esos ambientes híbridos, refiere al incremento en el acceso al conocimiento y mayor flexibilidad en dicho proceso.

consideramos que la situación de emergencia sanitaria sin precedentes que estamos atravesando, ha puesto al descubierto la necesidad de contar, desde las instituciones educativas, con plataformas y equipamiento que permitan y garanticen el desarrollo de estrategias de enseñanza-aprendizaje y evaluación de forma virtual y/o híbrida.

Por lo tanto, consideramos que la situación de emergencia sanitaria sin precedentes que estamos atravesando, ha puesto al descubierto la necesidad de contar, desde las instituciones educativas, con pla- 
taformas y equipamiento que permitan y garanticen el desarrollo de estrategias de enseñanza-aprendizaje y evaluación de forma virtual y/o híbrida. Además, se torna fundamental continuar contribuyendo desde el Estado (a través de políticas públicas como Conectar Igualdad por ejemplo) en la cobertura de dispositivos y conectividad para los sectores de estudiantes que en este momento se encuentran en desventajas sociales, económicas y habitacionales, teniendo en cuenta que estos factores pueden incidir en la permanencia y la promoción dentro del sistema educativo. 


\section{BIBLIOGRAFIA}

Area Moreira, Manual (19 de enero de 2015). "Los entornos de aprendizaje digitales". España, Universidad de la Laguna Ed. Disponible en: https://www.youtube.com/watch?time_continue=38\&v=iFpwzGJPMI8. Fecha de consulta: 10 de diciembre de 2018

- Goldar, María Rosa y equipo de cátedra (2019). “Programa de Trabajo Social III: Abordaje Comunitario y Grupal

- Desarrollo Local". Lic. en Trabajo Social, Facultad de Ciencias Políticas y Sociales. Mendoza, UNCuyo

- Goldar, María Rosa y equipo de cátedra (2020). “Programa de Trabajo Social III: Abordaje Comunitario y Grupal

- Desarrollo Local". Lic. en Trabajo Social, Facultad de Ciencias Políticas y Sociales. Mendoza, UNCuyo Osorio Gómez, Luz (2011). "Ambientes híbridos de aprendizaje". Actualidades Pedagógicas, N58. Julio-diciembre 2011, 29-44.

- Secretaría Académica (2020). “Protocolo para Mesas de exámenes finales en contexto de aislamiento social, preventivo y obligatorio". Facultad de Ciencias Políticas y Sociales. Mendoza, UNCuyo. Disponible en: http://fcp.uncuyo.edu.ar/ upload/protocolo-2020-propuesta-4-de-mayo.pdf 